\title{
TIME COURSE OF ATTENTIONAL MODULATIONS ON AUTOMATIC EMOTIONAL PROCESSING
}

\author{
Authors: Sonia Doallo, Fernando Cadaveira, Socorro Rodríguez Holguín
}

This is the peer reviewed version of the following article: Doallo S, Cadaveira F; Rodríguez Holguín S. (2007). Time course of attentional modulations on automatic emotional processing. Neuroscience Letters, 418, 111-116. doi:10.1016/j.neulet.2007.03.009. This article may be used for non-commercial purposes in accordance with Elsevier Terms and Conditions for Use of Self-Archived Versions 


\title{
Time course of attentional modulations on automatic emotional processing
}

\author{
Sonia Doallo, Fernando Cadaveira, Socorro Rodríguez Holguín \\ Department of Clinical Psychology and Psychobiology, University of Santiago de Compostela,
}

Corresponding author: Sonia Doallo. Faculty of Psychology. University of Santiago de Compostela Campus Sur, E-15782 Santiago de Compostela, Galicia, Spain. Tel.: +34 981563 100x13916; fax: +34981528071. E-mail address: sdoallo@usc.es .

\begin{abstract}
In a previous study using event-related potentials (ERPs) [S. Doallo, S. Rodríguez Holguín, F. Cadaveira, Attentional load affects automatic emotional processing: evidence from event-related potentials, Neuroreport 17 (2006) 1797-1801], we reported that differential responses to unattended peripheral affective pictures, as reflected by N1-P2 modulations at posterior regions, are modulated by attentional load at fixation. Here, new analyses of these data were performed to evaluate whether a sustained, broadly distributed, negative shift in the unattended pictures ERP waveforms, which displayed larger amplitudes for emotional stimuli, reflects an additional differential response to the emotional content. Under low-load conditions, unpleasant (versus neutral) pictures elicited greater negativities in the $80-140 \mathrm{~ms}$ latency range over frontocentral sites and more centroparietally distributed from 200 to $280 \mathrm{~ms}$. These findings provide further evidence of the time course of emotional processing at unattended locations and its modulation by attentional load.
\end{abstract}

\section{Keywords}

Event-related potentials; Attention; Emotion; Load; International affective picture system 


\section{Introduction}

An important issue on the relation between attention and emotion refers to the processing of emotional stimuli presented outside the focus of attention. Neuroimaging [14,20], lesion [19] and event-related potentials (ERPs) $[1,2,18]$ studies provide evidence for the capture of attention by emotional events. However, data indicating that the emotional value of task-irrelevant stimuli is processed when attention is actively engaged on other stimuli are not unequivocal. Some authors report that ERPs elicited by emotional stimuli are different from those triggered by neutral ones when they are attended, but not when they appear at task-irrelevant locations [6,8], suggesting that cortical stages of emotional processing are strongly modulated by focal attention. A recent ERP experiment [7] found that the extent to which the emotional processing is influenced by attention depends on factors such as foveal versus peripheral presentation. The critical role of the task-relevant attentional load on the processing of task-irrelevant emotional events has been also evidenced from functional magnetic resonance imaging studies [15,16]. In a previous report [5], we found ERP evidence consistent with the hypothesis that the differential brain response to unattended emotional stimuli depends strongly on the attentional load of the ongoing task. We recorded ERPs elicited by taskirrelevant unpleasant and neutral pictures briefly presented at peripheral locations while participants were actively monitoring the fixation ir order to perform a discrimination task varying in load (lowand high-load). With the aim of detecting any capture of attention by task-irrelevant unpleasant pictures, the $\mathrm{P} 2$ component was measured. $\mathrm{P} 2$ is specially sensitive to negative visual stimuli $[3,4]$ and it is also elicited in response to emotional stimuli outside the focus of attention [1]. Results revealed that unpleasant pictures (versus neutral pictures) produced enhanced N1-P2 amplitudes at parietoccipital and occipital sites only under low-load conditions. We concluded that the brain responses to the affective value of task-irrelevant peripheral pictures are modulated by the attentional load at fixation. Although these analyses were focused in the P2 component, grand-averaged waveforms also revealed that the ERP to unattended stimuli presented a sustained, broadly distributed, negative shift with larger amplitudes for emotional pictures than neutral pictures [5]. Here, we reanalyze our data with the aim of evaluating whether this difference reflects an additional differential response to the emotional content of the pictures and to investigate in more detail the time course of this effect.

\section{Methods}

Ten female volunteers $(21-30$ years old, mean $=26.4$, S.D. $=2.5)$ were assessed. All were righthanded, had normal or corrected-to-normal vision, and had no history of psychiatric or neurological disorders. Participants gave informed consent. 
The target stimuli were short $\left(0.6^{\circ} \times 2.3^{\circ}\right)$ or long $\left(0.6^{\circ} \times 3.4^{\circ}\right)$ vertical bars equiprobably flashed for $50 \mathrm{~ms}$ in the center of the display. Vertical bars were sequentially presented, according to a stimulus 1 (S1)-stimulus 2 (S2) paradigm. During the delay period between S1 and S2, 40 negative and 40 neutral pictures $(8 \circ \times 5.7 \circ)$ selected from the International Affective Picture System (IAPS) ${ }^{1}[11]$ were occasionally presented (negative $p=.15$, neutral $p=.15$ ) at $5.7^{\circ}$ to the left or to the right from a fixation cross for $50 \mathrm{~ms}$. The two categories significantly differed from each other in the valence rating $(2.1 \pm 0.6$ versus $5.2 \pm 0.3 ; t(39)=-26.314, p=.000)$ and in the arousal rating $(7.2 \pm 0.4$ versus $3.2 \pm 0.5 ; t(39)=38.901, p=.000)$ on the basis of the normative values of IAPS in the Spanish population [13]. Valence and arousal ratings of each picture are shown in Tables 3 and 4 in supplementary material online. Statistical comparisons confirmed that the two groups of pictures did not significantly differ in luminance or spatial frequency. The difficulty of the relevant central task was increased in a different experimental block, in which size discrimination was rendered difficult by reducing the difference between the bars (short bars: $0.6^{\circ} \times 2.3^{\circ}$; long bars: $0.6^{\circ} \times 2.5^{\circ}$ ). Participants were required to maintain eye fixation on the central cross, and to discriminate whether the second bar of the match (S2) was equal or different in size from the first bar (S1), pressing one button with one hand if equal in size and another button with the other hand if different in size, while ignoring the peripheral pictures. The assignment of the response hand was balanced across participants. The interval from $\mathrm{S} 1$ onset to distractor onset (SOA) randomly varied from 100 to $1100 \mathrm{~ms}$. SOA between $\mathrm{S} 1$ and S2 randomly varied from 1200 to $1500 \mathrm{~ms}$. The intertrial intervals randomly varied from 2000 to $2300 \mathrm{~ms}$. In order to familiarize the participants with the task, the low-load condition was presented first. Each experimental condition consisted of eight trial blocks. The maximum number of trials per block was 125 .

ERPs (bandpass $0.1-50 \mathrm{~Hz}, 500 \mathrm{~Hz} / \mathrm{channel}$ ) were recorded from 16 active electrodes (F3, F4, FC3, FC4, C3, C4, T5, T6, CP3, CP4, P3, P4, PO3, PO4, O1, and O2), referred to a nasal electrode. Vertical and horizontal eye movements were recorded bipolarly from above and below the left eye and from the outer canthus of each eye. Reaction time (RT) and percentage of correct responses were recorded for each trial. Data were collected over epochs from $60 \mathrm{~ms}$ before stimulus to $600 \mathrm{~ms}$ after stimulus. Trials with eye blinks or horizontal eye movements were rejected. For each participant, ERPs elicited by neutral and emotional distractors were averaged (digital bandpass of $0.1-30 \mathrm{~Hz}$ ) separately for each visual field and condition.

\footnotetext{
${ }^{1}$ The IAPS numbers for negative pictures were: 1050, 1300, 2120, 2800, 2900, 3000, 3010, 3030, 3051, 3053, $3060,3071,3100,3102,3120,3130,3150,3170,3230,3350,3400,3550,6212,6250,6260,6313,6510,6550$, 6560, 6570, 9040, 9050, 9400, 9410, 9421, 9520, 9630, 9800, 9810, 9920; for neutral pictures: 2190,2200 , $2210,2840,5500,5510,5520,5530,5531,5532,5533,5534,6150,7000,7002,7006,7009,7010,7025,7030$, 7034, 7035, 7040, 7050, 7060, 7080, 7090, 7150, 7170, 7190, 7207, 7217, 7224, 7233, 7235, 7490, 7500, 7550, 7820,7830 . The valence and arousal normative ratings for the Spanish population [13] are available in the web page: http://www.fedap.es/IberPsicologia/iberpsi5-1/iaps/iaps.pdf.
} 
Visual inspection of waveforms revealed that the ERP to distractor stimuli was affected by a sustained negative shift which showed larger amplitudes for emotional pictures than for neutral pictures. To evaluate whether this difference reflects a differential response to the emotional content, the mean voltage values within twelve latency windows of $20 \mathrm{~ms}$ each one, from 60 to $300 \mathrm{~ms}$, were analyzed. Repeated-measure ANOVAs were performed for each latency window with five within-subject factors: region (frontal / frontocentral / central / centroparietal / parietal / temporal / parietooccipital / occipital), hemisphere of recording (left / right), load condition (low / high), emotional content (neutral / negative) and visual field (left / right). Significance levels were determined using degrees of freedom after applying the Greenhouse-Geisser correction when appropiate. Post-hoc comparisons were performed using the Bonferroni adjustment for multiple comparisons.

The RT and accuracy data were subjected to repeated measures ANOVAs with load (two levels) and distractor (none/left neutral/right neutral/left emotional/right emotional, five levels) as factors.

\section{Results}

Behavioral performance confirmed that the central attentional load was effectively manipulated: (1) responses were slower to target bars preceded by irrelevant stimuli (neutral and emotional) than to those not preceded by distractors $(F(4,36)=3.329, p=.020)$. This interference effect was only significant in the low-load condition $(F(4,36)=2.998, p=.031)$; (2) hit rates were also lower during the high-load $(63.8 \pm 9.8)$ than low-load $(91.9 \pm 7.9)$ condition $(F(1,9)=351.622, p=.000)$.

ERPs mean amplitudes to neutral and emotional stimuli were significantly different during the 80-140 ms and 200-280 ms post-stimulus intervals, with larger negative amplitudes to unpleasant stimuli than to neutral stimuli (all $F(1,9)>1.367$, all $p<.046$ ). To further explore the time course and the topographical distribution of these differences, and based on statistically significant interactions between emotional content, region and load in these two time ranges (all $F(7,63)>3.242$, all $p<$ .019 ), additional analyses were conducted on 20-ms time windows, separately for each experimental condition and region (see Tables 1 and 2).

\section{Tables 1 and 2}

For the low-load condition, the effects of the emotional content of the pictures were observed in the first latency windows $(80-140 \mathrm{~ms}$ ) with a predominantly frontocentral distribution (all $F(1,9)>5.814$, all $p<.039$ ), with larger negative amplitudes to unpleasant pictures than neutral pictures. Effects of emotional content were also present over more posterior sites in the 120-140 ms latency range (all $F(1,9)>5.213$, all $p<.048)$. From 80 to $120 \mathrm{~ms}$, the effect on these posterior electrodes was not so 
marked, although there were emotional content $\times$ visual field (all $F(1,9)>5.445$, all $p<.045)$ and emo- tional content $\times$ hemisphere $\times$ visual field $($ all $F(1,9)>5.559$, all $p<.043)$ interactions. At the later interval (200-280 ms), the differential response to the emotional content had a more centroparietal distribution (all $F(1,9)>8.522$, all $p<.017)$.

For the high-load condition, the effects of the emotional content were modulated by the hemisphere of recording and the visual field (all $F(1,9)>5.228$, all $p<.048$ ). There were no reliable emotional content effects at any region or hemisphere.

Figs. 1 and 2 show the ERPs to unpleasant and neutral pictures in the low-load condition, for the most relevant scalp locations.

Figure 1 and 2

\section{Discussion}

In a previous report [5], differential brain responses to task-irrelevant emotionally salient stimuli, as reflected by amplitude modulations of the N1-P2 component at parietoccipital and occipital regions, were observed when attention was actively engaged in a low-load primary task, but not when the load was increased. We concluded that unpleasant stimuli receive prioritized processing compared with neutral stimuli even when they are presented outside the attentional focus, and that this processing is strongly attenuated by the attentional load of the ongoing task. Here, we performed new analyses of our ERP data, to evaluate whether a sustained negative shift, present in the distractor pictures ERP waveforms, broadly distributed and which showed a larger amplitude for emotional stimuli, might indicate an additional differential brain response to the emotional value of stimuli. These new analyses revealed that unpleasant pictures produced greater negativities, with a predominantly frontocentral distribution, from 80 to $140 \mathrm{~ms}$ post-stimulus, and more centroparietally distributed from 200 to $280 \mathrm{~ms}$. Again, these effects were only significant in the low-load condition. Taken together, these findings confirm that attentional capture by emotionally salient events depends on the availability of attentional resources.

The early differential neural response (80-140 ms) to the emotional content of stimuli at frontocentral locations might reflect the activation of the prefrontal cortex, which has been involved in the fast categorization of emotion (see below). An early ERP differentiation between pleasant and unpleasant stimuli (facial expressions) has been also reported by previous studies [17] during passive observation conditions. Although others $[6,8]$ have not found ERP emotional modulations under unattended 
conditions, noticeable differences in the experimental designs might explain the discrepancies (see [5] for a further discussion).

The early effect of emotional content is also consistent with studies reporting an electrophysiological frontal response to unpleasant stimuli in short latencies [1,2,9]. Kawasaki et al. [9] recorded differential neural responses to negative visual stimuli in the 120-170 ms latency range at ventromedial prefrontal areas (see also [10]). These authors suggested that the prefrontal circuits might provide a fast categorization of emotion, which might modulate the processing of visual information in other brain regions. In a similar vein, Carretie' et al. [2] observed that unconsciously perceived signals of threat (versus nonnegative pictures) produced a larger activation in the ventromedial prefrontal cortex (VMPFC) around $150 \mathrm{~ms}$. They proposed that the VMPFC is involved in the top-down regulation of attention, increasing the subsequent visual cortex activation, and resulting in a facilitation of danger processing.

In line with these proposals, the early differential emotional response over anterior regions observed in the present study, might be considered as a correlate of the involvement of the prefrontal cortex in a mechanism of top-down facilitation of the visual processing system. The subsequent significant ERP modulations, described in our previous work [5], at the level of N1-P2 component over the posterior scalp region, might reflect the result of these modulatory effects of the prefrontal cortex on visual regions. In addition, the present findings allow to appreciate that these early effects of emotional content on ERPs are influenced by the availability of attentional resources.

Emotional modulations of the ERPs from 200 to 280 ms post-stimulus, centroparietally distributed, under low-load conditions, were also found. ERP amplitude modulations in this latency range, with larger negativities to unpleasant stimuli, have been reported in several recent studies. Liddell et al. [12] found larger amplitudes of the N2 component, with a central distribution, in response to the subliminal presentation of fearful faces as compared with neutral ones. Schupp et al. [18] found an affective discrimination of IAPS pictures (reflected by larger negativities to affective pictures at temporo-occipital sites) when the top-down attentional control was directed to a primary task. The methodological differences between studies make difficult a detailed comparison between results or an explanation of the topographical distribution differences. In spite of this, the present findings provide converging evidence supporting an automatic response to emotional stimuli, either faces [12] or IAPS pictures [18], which does not depend of their explicit categorization and that can be present when attention is focused in a main task [18]. The present data extend these findings indicating that the electrophysiological response to the affective value of task-irrelevant IAPS pictures is strongly influenced by the availability of resources of attention. Even though no definite conclusions can be extracted from these data because of the small sample size, the results appear to provide new electrophysiological support for the hypothesis that attentional load of the ongoing task may be 
crucial in determining the extent in which task-irrelevant emotional stimuli are processed $[15,16]$. There is another methodological limitation that should be noted. Only negative and neutral stimuli were employed, which make it difficult to distinguish the contributions to the observed effects of valence and arousal. Additional experiments including also arousing pleasant pictures are necessary to reach strong conclusions on automatic attention to task-irrelevant affective pictures as a function of their two basic dimensions.

In conclusion, the present findings provide evidence supporting that the prioritized processing of stimuli with emotional value presented at task-irrelevant locations strongly depends on the attentional load at relevant locations. They also reveal that the time course of emotional value processing of unattended stimuli is characterized by an early (80-140 ms) modulation of the frontal electrophysiological response which precedes the differential processing at posterior cortical regions.

\section{Acknowledgements}

This study was supported by Spain's Ministerio de Educación y Ciencia (SEJ2004-01377) and Xunta de Galicia (PGIDT05PXI21101PN). The authors thank to Dr. S. Bará (Optics Area, Applied Physics Department at the University of Santiago de Compostela) for methodological assistance.

\section{Supplementary data}

Supplementary data associated with this article can be found, in the online version

\section{References}

[1] L. Carretié, J.A. Hinojosa, M. Martín-Loeches, F. Mercado, M. Tapia, Automatic attention to emotional stimuli: neural correlates, Hum. Brain Mapp. 22 (2004) 290-299.

[2] L. Carretié, J.A. Hinojosa, F. Mercado, M. Tapia, Cortical response to subjectively unconscious danger, Neuroimage 24 (2005) 615-623.

[3] L. Carretié, M. Martín-Loeches, J.A. Hinojosa, F. Mercado, Emotion and attention interaction studied through event-related potentials, J. Cogn. Neurosci. 13 (2001) 1109-1128. 
[4] L. Carretié, F. Mercado, M. Tapia, J.A. Hinojosa, Emotion, attention, and the 'negativity bias', studied through event-related potentials, Int. J. Psychophysiol. 41 (2001) 75-85.

[5] S. Doallo, S. Rodríguez Holguín, F. Cadaveira, Attentional load affects automatic emotional processing: evidence from event-related potentials, Neuroreport 17 (2006) 1797-1801.

[6] M. Eimer, A. Holmes, F.P. McGone, The role of spatial attention in the processing of facial expression: an ERP study of rapid brain responses to six basic emotions, Cogn. Affect. Behav. Neurosci. 3 (2003) 97-110.

[7] A. Holmes, M. Kiss, M. Eimer, Attention modulates the processing of emotional expression triggered by foveal faces, Neurosci. Lett. 394 (2006) 48-52.

[8] A. Holmes, P. Vuilleumier, M. Eimer, The processing of emotional facial expression is gated by spatial attention: evidence from event-related brain potentials, Cogn. Brain Res. 16 (2003) 174-184.

[9] H. Kawasaki, R. Adolphs, O. Kaufman, H. Damasio, A.R. Damasio, M. Granner, H. Bakken, T. Hori, M.A. Howard 3rd, Single-neuron responses to emotional visual stimuli recorded in human ventral prefrontal cortex, Nat. Neurosci. 4 (2001) 15-16.

[10] H. Kawasaki, R. Adolphs, H. Oya, C. Kovach, H. Damasio, O. Kaufman, M. Howard 3rd, Analysis of single-unit responses to emotional scenes in human ventromedial prefrontal cortex, J. Cogn. Neurosci. 17 (2005) 1509-1518.

[11] P.J. Lang, M.M. Bradley, B.N. Cuthbert, International Affective Picture System (IAPS): Instruction Manual and Affective Ratings, The Center for Research in Psychophysiology, University of Florida, 1999.

[12] B.J. Liddell, L.M. Williams, J. Rathjen, H. Shevrin, E. Gordon, A temporal dissociation of subliminal versus supraliminal fear perception: an event-related potential study, J. Cogn. Neurosci. 16 (2004) 479-486.

[13] J. Moltó , S. Montañés, R. Poy, P. Segarra, M.C. Pastor, M.P. Tormo, I. Ramírez, M.A. Hernández, M. Sánchez, M.C. Fernández, J. Vila, Un nuevo metodo para el estudio experimental de las emociones: el International Affective Picture System (IAPS). Adaptación española [A new method for the experimental study of emotions: The International Affective Picture System (IAPS)], Rev. Psicol. Gen. Aplicada 52 (1999) 55-87.

[14] A. Öhman, Automaticity and the amygdala: nonconscious responses to emotional faces, Curr. Dir. Psychol. Sci. 11 (2002) 62-66. 
[15] L. Pessoa, S. Kastner, L.G. Ungerleider, Attentional control of the processing of neutral and emotional stimuli, Cogn. Brain Res. 15 (2002) 31-45.

[16] L. Pessoa, S. Padmala, T. Morland, Fate of unattended fearful faces in the amygdala is determined by both attentional resources and cognitive modulation, Neuroimage 28 (2005) 249-255

[17] D. Pizzagalli, M. Regard, D. Lehmann, Rapid emotional face processing in the human right and left brain hemispheres: an ERP study, Neuroreport 10 (1999) 2691-2698.

[18] H.T. Schupp, M. Jungho“ fer, A.I. Weike, A.O. Hamm, Attention and emotion: an ERP analysis of facilitated emotional stimulus processing, Neuroreport 14 (2003) 1107-1110.

[19] P. Vuilleumier, Facial expression and selective attention, Curr. Opin. Psychiatry 15 (2002) 291300 .

[20] P. Vuilleumier, J.L. Armony, J. Driver, R.J. Dolan, Effects of attention and emotion on face processing in the human brain: an event-related fMRI study, Neuron 30 (2001) 829-841. 


\section{Tables}

Table 1. Time course of emotional content effects ( $p$-values) in the low-load condition

\begin{tabular}{|c|c|c|c|c|c|c|c|}
\hline \multirow[b]{3}{*}{ Region } & \multicolumn{7}{|c|}{ LOW-LOAD } \\
\hline & \multicolumn{7}{|c|}{ Latency windows (ms) } \\
\hline & $80-100$ & $100-120$ & $120-140$ & 200-220 & $220-240$ & $240-260$ & $260-280$ \\
\hline $\mathbf{F}$ & $\begin{array}{c}\mathrm{E} \\
.023\end{array}$ & & & & & & \\
\hline FC & $\begin{array}{c}\mathrm{E} \\
.014\end{array}$ & $\begin{array}{c}\mathrm{E} \\
.028\end{array}$ & $\begin{array}{c}\mathrm{E} \\
.030\end{array}$ & $\begin{array}{c}\mathrm{E} \\
.041\end{array}$ & & & $\begin{array}{c}\mathrm{E} \\
.025\end{array}$ \\
\hline C & $\begin{array}{c}\mathrm{E} \\
.039 \\
\end{array}$ & $\begin{array}{c}\mathrm{E} \\
.029 \\
\end{array}$ & $\begin{array}{c}\mathrm{E} \\
.021 \\
\end{array}$ & $\begin{array}{c}\mathrm{E} \\
.002 \\
\end{array}$ & & $\begin{array}{c}\mathrm{E} \\
.013 \\
\end{array}$ & $\begin{array}{c}\mathrm{E} \\
.011 \\
\end{array}$ \\
\hline $\mathbf{C P}$ & & $\begin{array}{c}\mathrm{E} \\
\mathrm{E} \times \mathrm{H} \times \mathrm{H} \mathrm{VF} \\
.043 \\
\end{array}$ & $\begin{array}{c}\mathrm{E} \\
.019\end{array}$ & $\begin{array}{c}\mathrm{E} \\
.002\end{array}$ & $\begin{array}{c}\mathrm{E} \\
.017\end{array}$ & $\begin{array}{c}\mathrm{E} \\
.009\end{array}$ & $\begin{array}{c}\mathrm{E} \\
.011\end{array}$ \\
\hline $\mathbf{P}$ & & $\begin{array}{c}\mathrm{E} \times \mathrm{H} \times \mathrm{VF} \\
.038\end{array}$ & $\begin{array}{c}\mathrm{E} \\
.026 \\
\end{array}$ & $\begin{array}{c}\mathrm{E} \\
.037 \\
\end{array}$ & & $\begin{array}{c}\mathrm{E} \\
.038 \\
\end{array}$ & $\begin{array}{c}\mathrm{E} \\
.039 \\
\end{array}$ \\
\hline PO & & $\begin{array}{c}\mathrm{E} \times \mathrm{VF} \\
.045 \\
\mathrm{E} \times \mathrm{H} \times \mathrm{VF} \\
.027\end{array}$ & $\begin{array}{c}\mathrm{E} \\
.035 \\
\end{array}$ & & & & $\begin{array}{c}\mathrm{E} \times \mathrm{H} \times \mathrm{VF} \\
.001\end{array}$ \\
\hline $\mathbf{O}$ & $\begin{array}{c}\text { Ex VF } \\
.035\end{array}$ & $\begin{array}{c}\text { E x VF } \\
.023\end{array}$ & $\begin{array}{c}\mathrm{E} \\
.048\end{array}$ & & & & $\begin{array}{c}\mathrm{E} \times \mathrm{H} \times \mathrm{VF} \\
.026\end{array}$ \\
\hline $\mathbf{T}$ & $\begin{array}{c}\mathrm{E} \times \mathrm{VF} \\
.008\end{array}$ & $\begin{array}{c}\text { E x VF } \\
.036\end{array}$ & & & & & \\
\hline
\end{tabular}

H: hemisphere; E: emotional content; VF: visual field, F: frontal, FC: frontocentral, C: central, CP: centroparietal, P: parietal, PO: parietoccipital, O: occipital, T: temporal. The significant main effects of the factor emotional content are presented in shaded.

Table 2. Time course of emotional content effects ( $p$-values) in the high-load condition

\begin{tabular}{|c|c|c|c|c|c|c|c|}
\hline \multirow[b]{3}{*}{ Region } & \multicolumn{7}{|c|}{ HIGH-LOAD } \\
\hline & \multicolumn{7}{|c|}{ Latency windows (ms) } \\
\hline & $80-100$ & $100-120$ & $120-140$ & $200-220$ & $220-240$ & $240-260$ & 260-280 \\
\hline \multicolumn{8}{|l|}{$\mathbf{F}$} \\
\hline \multicolumn{8}{|l|}{ FC } \\
\hline \multicolumn{8}{|l|}{ C } \\
\hline CP & $\begin{array}{c}\mathrm{E} \times \mathrm{H} \times \mathrm{VF} \\
.029\end{array}$ & & & & & & \\
\hline $\mathbf{P}$ & $\begin{array}{c}\mathrm{E} \times \mathrm{H} \times \mathrm{VF} \\
.012\end{array}$ & $\begin{array}{c}\mathrm{E} \times \mathrm{H} \times \mathrm{VF} \\
.037\end{array}$ & & & & & $\begin{array}{c}\mathrm{E} \times \mathrm{H} \times \mathrm{VF} \\
.034\end{array}$ \\
\hline PO & $\begin{array}{c}\mathrm{E} \times \mathrm{H} \times \mathrm{VF} \\
.017\end{array}$ & & & & & & \\
\hline $\mathbf{O}$ & $\begin{array}{c}\mathrm{E} \times \mathrm{H} \times \mathrm{VF} \\
.042\end{array}$ & $\begin{array}{c}\mathrm{E} \times \mathrm{H} \times \mathrm{VF} \\
.048\end{array}$ & & & & & \\
\hline $\mathbf{T}$ & $\begin{array}{c}\mathrm{H} \times \mathrm{E} \\
.024 \\
\mathrm{E} \times \mathrm{H} \times \mathrm{VF} \\
.008\end{array}$ & $\begin{array}{c}\mathrm{E} \times \mathrm{H} \times \mathrm{VF} \\
.012\end{array}$ & & & $\begin{array}{c}\mathrm{E} \times \mathrm{H} \times \mathrm{VF} \\
.001\end{array}$ & & $\begin{array}{c}\mathrm{H} \times \mathrm{E} \\
.033\end{array}$ \\
\hline
\end{tabular}

H: hemisphere; E: emotional content; VF: visual field, F: frontal, FC: frontocentral, C: central, CP: centroparietal, P: parietal, PO: parietoccipital, O: occipital, T: temporal. 
Figures

LOW-LOAD CONDITION: Distractors at the LVF
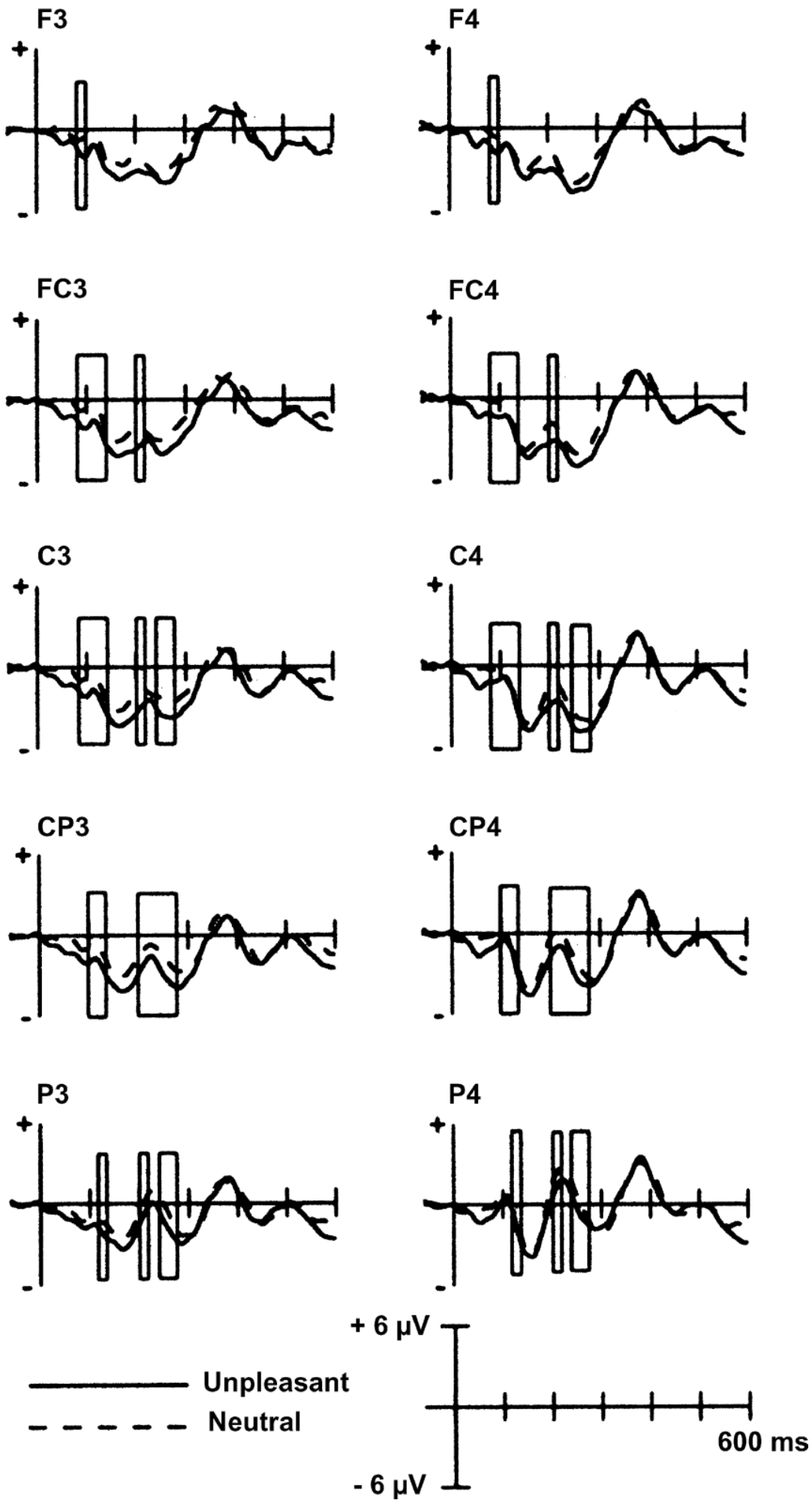

Figure 1. Low-load condition: grand-averaged ERPs for unpleasant and neutral task-irrelevant pictures at the left visual field (LVF). The boxes indicate the time intervals where the effect of emotional content was significant. 

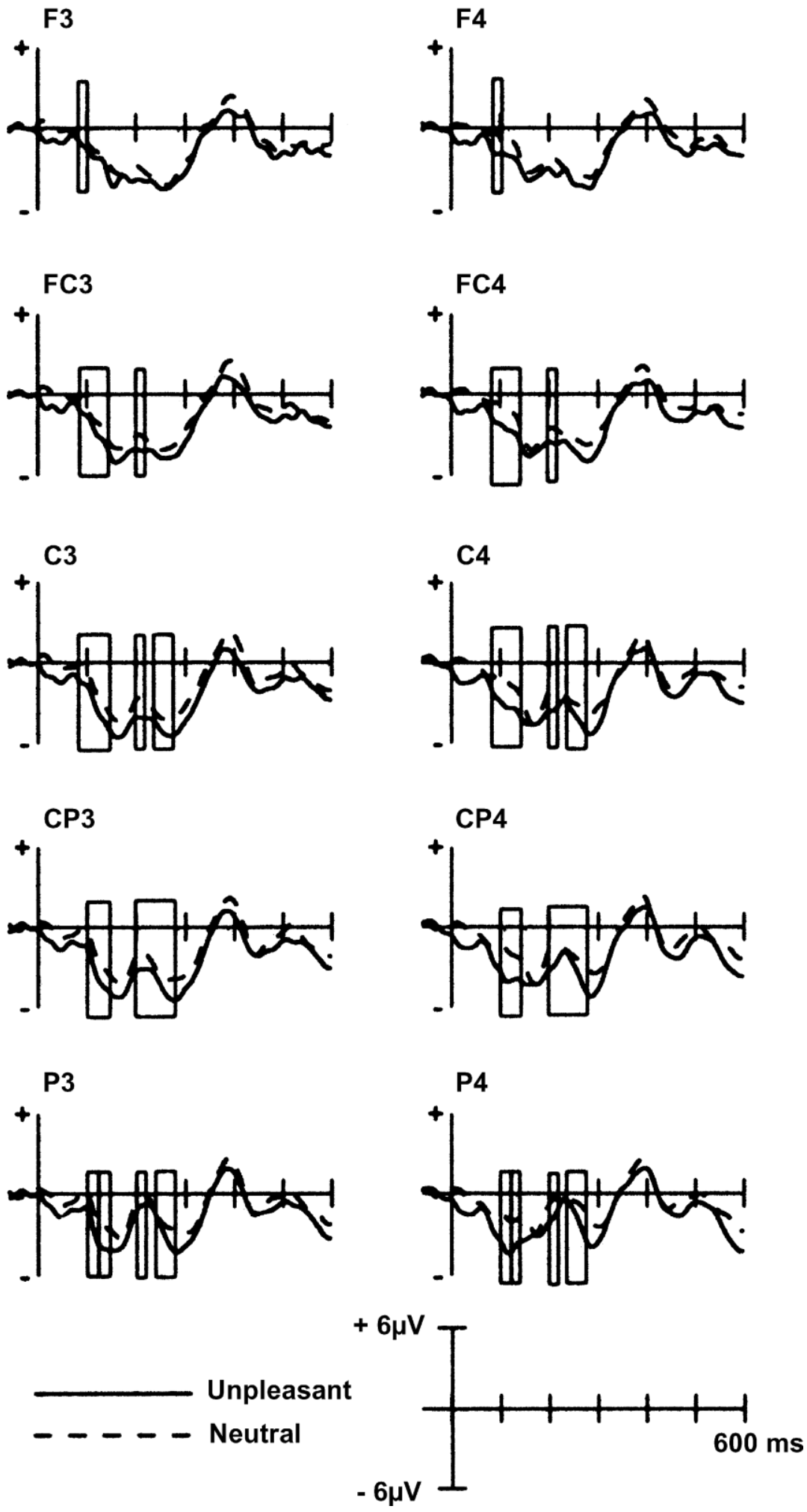

Figure 2. Low-load condition: grand-averaged ERPs for unpleasant and neutral task-irrelevant pictures at the right visual field (RVF). The boxes indicate the time intervals where the effect of emotional content was significant. 